\title{
Hot topics from the Assemblies
}

Results of initial low-dose computed tomographic screening for lung cancer.

Authors: Church TR, Black WC, Aberle DR, et al.

N EnglJ Med 2013; DOI: 10.1056/NEJMoa1209120

Summary: Screening with low-dose helical computed tomography (CT) has been shown to reduce lung cancer mortality more than chest radiography. Using data from 53,439 participants (55-74 years old with a history of at least 30 pack-years of smoking), the screening, diagnosis and treatment results from the initial round of screening in the National Lung Screening Trial (NLST) were assessed. $27.3 \%$ of patients in the CT screening group and $9.2 \%$ in the radiography screening group had a positive screening result and $>90 \%$ in each group had thereafter additional follow-up diagnostic procedures such as imaging and surgery. Lung cancer was diagnosed in $1.1 \%$ in the low-dose CT group (54\% in stage I, $41.1 \%$ in stages II-IV) versus $0.7 \%$ in the radiography group $36.8 \%$ in stage I, $58.9 \%$ in stages II-IV). Specificity and sensitivity for low-dose CT were $73.4 \%$ and $93.8 \%$, and were $91.3 \%$ and $73.5 \%$ for chest radiography, respectively.

Discussion: Screening with low-dose chest CT scan can achieve earlier diagnosis of lung cancer. Whether this screening method can reduce lung cancer mortality is still to be proved and therefore new data are awaited.

Reviewed by: Georgia Hardavella (Greece, Assembly 11)

Being overweight increases susceptibility to indoor pollutants among urban children with asthma. Authors: Lu KD, Breysse PN, Diette GB, et al.

J Allergy Clin Immunol 2013; DOI: 10.1016/j. jaci.2012.12.1570.

Summary: The increasing prevalence of asthma has been most pronounced in westernised countries, and has paralleled that of obesity. Some of the highest prevalence estimates for asthma have been found in lowincome urban settings, where obesity is prevalent and air pollution levels are high. The present study examined the possibility of interactions between weight and air pollution on asthma health in children. A cohort of 148 non-smoking children (aged 5-17 years) with persistent asthma was followed for one year. Asthma symptoms, lung function, healthcare use and levels of bedroom pollutants were measured every three months. Of the participants, 91\% were African American and 64\% reported a household income $<$ US $\$ 30,000$ per year. In the year before enrolment, $82 \%$ had had an emergency visit for asthma and $20 \%$ had been hospitalised. Obesity was seen in $28 \%$, and $16 \%$ were overweight. Nocturnal asthma symptoms $(p=0.02)$ and exercise-induced symptoms $(p=0.07)$ tended to be more common in overweight and obese children. Levels of fine particle matter (PM2.5) and nitric oxide, but not coarse particles (PM2.5-10) were associated with an increased risk of several asthma symptoms in overweight or obese children but not in normal weight children.

Discussion: The present study demonstrates positive interactions between overweight/obesity and air pollution on asthma symptoms. This is one likely explanation for the poor asthma control commonly seen in low-income urban settings. However, the results do not explain the high asthma prevalence in these areas, and future studies should target incidence of asthma in relation to obesity and air pollution.

Reviewed by: Anders Bjerg (Sweden, Assembly 6)

Effectiveness and predictors of success of noninvasive ventilation during $\mathrm{H}_{1} \mathrm{~N}_{1}$ pandemics: a multicenter study. Authors: Nicolini A, Tonveronachi E, Navalesi P, et al.

Minerva Anestesiol 2012; 78:1333-1340

Summary In this investigation, treatment of hypoxaemic subjects with $\mathrm{H}_{1} \mathrm{~N}_{1}$ influenza is described. It is a prospective multicentre study describing the utility of noninvasive ventilation (NIV) in patients with acute respiratory failure caused by $\mathrm{H}_{1} \mathrm{~N}_{1}$ virus infection confirmed by rapid screening test, and $\mathrm{PaO}_{2} / \mathrm{FiO}_{2}<300$. NIV was aimed in 98 treated patients, but in 38 subjects immediate intubation was required. Out of the remaining 60 patients, 47 were successfully ventilated whereas 13 had to be intubated due to nontolerated or unsuccessful ventilation, described as clinical deterioration or failure to maintain $\mathrm{PaO}_{2} / \mathrm{FiO}_{2}>110$. The authors describe in detail NIV titration and interfaces. Patient factors predictive for NIV failure were: higher Simplified Acute Physiology Score II (SAPS II), lower $\mathrm{PaO}_{2} / \mathrm{FiO}_{2}$ ratio at admission, and a higher number of comorbidities. NIV was effective in preventing endotracheal intubation in $48 \%$ of the patients, and in $75 \%$ of patients not requiring immediate intubation. Hospital mortality was significantly higher in those patients who failed on NIV than in those who succeeded, probably because some of the treated patients were initially too sick for NIV.

Discussion NIV may be considered a good treatment option in developing acute hypoxaemic respiratory insufficiency, in selected patients with $\mathrm{H}_{1} \mathrm{~N}_{1}$ infection, especially if there is a shortage of intensive care beds. Reviewed by: Szymon Skoczynski (Poland, Assembly 2) and Stylianos Orfanos (Greece, Assembly 2)

Clinical measures of disease in adult non-CF bronchiectasis correlate with airway microbiota composition.

Authors: Rogers GB, van der Gast C), Cuthbertson L, et al.

Thorax 2013; DOI: 10.1136/thoraxjnl-2012-203105.

Summary Bacterial colonisation of the airways can increase the risk of exacerbations in patients with bronchiectasis, as well as playing a role in disease progression. This study examined samples from the lower airways of patients with non-CF bronchiectasis to determine the bacterial community composition. The study used a cross-sectional design with culture independent molecular techniques. 41 patients with non-CF bronchiectasis were studied with induced sputum and bronchiolar lavage (BAL). DNA extracted from samples was analysed using 165 rDNA gene 454 pyrosequencing and bacteria species were then 
separated into either "core" bacterial groups (abundant bacteria found commonly across patients) or "satellite" bacterial groups (rarer bacteria that are of lower abundance). Bronchiectasis clinical markers relate to the characteristics of the microbiota. 140 strains of bacteria were identified within the patient cohort. The core group, included Pseudomonas aeruginosa, Haemophilus influenzae, Streptococcus Pneumoniae, Veillonella, Prevotella, and Neisseria spp. The diversity of bacteria had a direct relationship with FEV1. The bacterial community diversity and composition had a significant relationship with patient's $\mathrm{FEV}_{1}$, neutrophil count and Leicester cough score.

Discussion: In-depth examination of a patient's airway bacterial community could give an indication of their disease severity. A better understanding of the importance of community diversity as well as individual pathogens will be needed to better target therapy in the future.

Reviewed by: Lucy Poppelwell (UK, Assembly 10)

Tiotropium Respimat Inhaler and the Risk of Death in COPD.

Authors: Wise RA, Anzueto A, Cotton D, et al.

N EnglJ Med 2013; DOI: 10.1056/NEJMoa1303342.

Summary: Previous meta-analyses have highlighted a potential increase in cardiovascular and all-cause mortality of chronic obstructive pulmonary disease (COPD) associated with Tiotropium Soft Mist Inhaler (SMI, Respimat) compared with Tiotropium Handihaler or placebo. TIOSPIR was a double-blind, double-dummy, randomised clinical trial, with a study population of 17135 patients, which was conducted to compare the efficacy and safety of Tiotropium Respimat versus Handihaler. During a mean follow up of 2.3 years, there were 1302 deaths, which were similarly distributed among the study groups (SMI $2.5 \mu \mathrm{g}, 5 \mu \mathrm{g}$ and Handihaler $18 \mu \mathrm{g}$ ) and indicated non-inferiority with regards to all-cause mortality. The effect estimates of SMI compared with Handihaler at both doses examined were: $2.5 \mu \mathrm{g}: 1.00$ (95\% Cl 0.87-1.14) and $5 \mu \mathrm{g}: 0.96$ (95\% Cl 0.84-1.09). No difference was highlighted in any of the other measurements, including risk of first exacerbation, time to first exacerbation, trough $\mathrm{FEV}_{1}$ and side effects.
Discussion: TIOSPIR provides evidence that Respimat and Handihaler have comparable safety and efficacy. Further studies are needed, as patients with unstable cardiac conditions, who are at higher risk of cardiovascular mortality were excluded from this study. It remains unclear why "real life" data continue to demonstrate an increase in cardiovascular mortality with Tiotropium Respimat.

Reviewed by: Alexandros Mathioudakis (Greece, Assembly 5)

Racemic adrenaline and inhalation strategies in acute bronchiolitis.

Authors: Skjerven HO, Hunderi JO, Brugmann-Pieper SK, et al.

N EnglJ Med 2013; DOI: 10.1056/NEJMoa1301839.

Summary: Bronchiolitis is common in infants, frequently leading to hospitalisation. Currently there is no evidence for use of inhaled adrenaline in these children. In a multicentre factorial ( 2 by 2 ) randomised clinical trial, the authors compared the effects of: 1) inhaled adrenaline versus inhaled saline; and 2) on-demand versus fixed schedule inhalations. In total, 404 infants $<1$ year old admitted with bronchiolitis were included. The primary outcome was "length of hospital stay", which was on average 80 hours for all included infants. The results convincingly show there is no benefit of inhaled adrenaline compared to inhaled saline. The ondemand inhalation schedule did lead to a significantly shorter hospital stay compared to the fixed schedule. The average difference of 13 hours can be considered a clinically important improvement. It should be realised that the intervention on inhalation schedule was not blinded. Nevertheless, significant improvement was also found for the more objective secondary outcomes of need for ventilatory support, oxygen therapy and nasogastric-tube feeding.

Discussion: It is well-conducted study with clinically relevant results. Unfortunately, translation of results to clinical practice is complicated by the fact that little information is given on timing and frequency of inhalations in both the fixed schedule and the on-demand group.

Reviewed by: Daan Caudri (The Netherlands, Assembly 7) 